\title{
Diseño ergonómico de aulas universitarias que permitan optimizar el confort y reducir la fatiga de estudiantes y docentes
}

\section{RESUMEN}

La presente investigación se realizó con la finalidad de conocer los aspectos ergonómicos (postura, mobiliario, condiciones ambientales y seguridad) que afectan la comodidad y fatiga de docentes y estudiantes en el aula universitaria. Se encontró que la postura inclinada, el mueble del computador, el ruido y la silla fueron los aspectos ergonómicos que más incomodan a docentes mientras que para los estudiantes la incomodidad se manifiesto, específicamente en el material del asiento, la forma y el material del respaldar.

Los resultados ameritan considerar nuevas aulas con diseño ergonómico ajustable a las dimensiones antropométricas de la población docente y estudiantil.

Palabras clave: antropometría, comodidad, diseño ergonómico, fatiga

ERGONOMIC DESIGN FOR UNIVERSITY CLASSROOMS TO ALLOW COMFORT AND REDUCTION OF FATIGUE IN STUDENTS AND TEACHERS

\section{ABSTRACT}

This research was conducted in order to meet the ergonomic aspects (posture, furnishings, environmental conditions and security) that affect comfort and fatigue of teachers and students in the university classroom. It was found that stooped posture, computer furniture, noise and chair were the ergonomic aspects that bother teachers while, students discomfort was manifested specifically the seat material, shape and material support.

These results merit considering new classrooms with ergonomic design adjustable to the anthropometric dimensions of the teaching equipment and student population.

Keywords: anthropometry, comfort, ergonomic design, fatigue

\section{INTRODUCCIÓN}

La investigación se realizó por el interés de brindar condiciones de trabajo favorables en donde la comodidad pueda ayudar al docente y al estudiante a mejorar el proceso de enseñanza aprendizaje y también a su calidad de vida.

La búsqueda de muebles resistentes y de buen aspecto e incluso el uso de la tecnología ha propiciado que se diseñen muebles que satisfagan estas exigencias y no así las medidas antropométricas de los usuarios y la funcionalidad para la ejecución de su tarea, provocando posturas forzadas, trastornos musculo esqueléticos, estrés y fatiga en sus usuarios.

\subsection{Objetivo General}

Proponer las condiciones ergonómicas en el diseño del aula universitaria para brindar comodidad a docentes y estudiantes, y reducir la fatiga.

\subsection{Objetivos Específicos}

a) Determinar los aspectos ergonómicos que influyen en la incomodidad y/o fatiga de docentes y estudiantes en el aula universitaria.

b) Conocer las medidas antropométricas de la población de docentes y estudiantes para saber si estas guardan relación con el mobiliario existente.

\section{MARCO TEÓRICO}

\subsection{Antecedentes del problema}

Los desarrollos de la medicina social, demuestran la intrínseca relación entre medio ambiente de trabajo y salud. Una de ellas señalada en un artículo por Esteve, J.M. (2005) "la salud de los docentes depende de las condiciones de trabajo de los mismos; por tanto, conforme cambian las condiciones de trabajo, es previsible esperar cambios en los indicadores de salud docente."

Según Vives (2005) "las investigaciones que existen de esta problemática enfatizan en la importancia de los factores

* Rosario Párraga Velásquez, Profesora Asociada del Departamento Académico de Producción y Gestión Industrial de la Facultad de Ingeniería Industrial. E-mail: mparragav@unmsm.edu.pe

* Teonila García Zapata, Profesora Principal Departamento Académico de Producción y Gestión Industrial de la Facultad de Ingeniería Industria de la Facultad de Ingeniería Industrial, asesora de tesis. E-mail: tgarciaz@unmsm.edu.pe 
contextuales, por ser estos los que generan mayor desconcierto e impotencia por estar fuera del radio de intervención individual de un docente", señala como indicador en primer orden de importancia un aula adecuada tanto para el docente como para el estudiante a fin de facilitar la tarea que se lleva en ella, que es la de formación del estudiante.

\subsection{El análisis ergonómico del puesto de trabajo}

La selección de los métodos ergonómicos por aplicar dependerá de los hallazgos encontrados en la estación/puesto de trabajo, en este caso el puesto de trabajo del docente y del estudiante. Para esta investigación se han resumido en los criterios de evaluación ergonómica que resultaron de una primera exploración y son los relativos a originar incomodidad y fatiga siendo los siguientes:

- Puesto de trabajo (mobiliario).

- Postura de trabajo.

- Condiciones de trabajo: Iluminación, Ruido y Ambiente térmico.

- Seguridad.

\subsection{Diseño del puesto y antropometría}

El diseñador debe tratar de proponer un diseño que satisfaga a la mayoría de la población de usuarios y no a todos por el costo que esto implica, para lo cual es fundamental la medida del hombre es decir la antropometría. El problema está en definir "la mayoría", pero en general, ésta se ha definido como el $90 \%, 95 \%$ de la población, para lo cual se ordenan los datos antropométricos con el uso del percentil.

\subsection{Aplicaciones}

El análisis ergonómico facilita a través de sus pautas la comprensión de todos los elementos que participan en la dinámica de un aula universitaria y las limitaciones de sus usuarios (docentes y estudiantes) para así determinar los factores a considerar en el espacio de trabajo que propicien la implementación de aulas cómodas para la producción y adquisición de conocimiento. GómezCano, V. (2007) hace referencia que existe una relación entre diseño ergonómico, ruido y productividad y es esencial entender la importancia de las limitaciones y capacidades del hombre para contribuir al aumento de la productividad y del bienestar de los mismos.

La justificación práctica se evidencia en el hecho de la necesidad de identificar y los factores que provocan incomodidad y fatiga en los usuarios y corregir las deficiencias encontradas en las aulas de forma que se pueda diseñar mobiliario adecuado que ayude a mejorar el rendimiento del estudiante y del docente buscando además el bienestar social.

\section{FORMULACIÓN DEL PROBLEMA}

¿Qué condiciones ergonómicas en el diseño del aula universitaria afectan la comodidad y producen fatiga en docentes y estudiantes?

\section{Problemas específicos:}

1. ¿Qué aspectos ergonómicos influyen en la comodidad y/o fatiga de docentes y estudiantes en el aula universitaria?

2. ¿Las medidas del mobiliario guardan relación con las medidas antropométricas de docentes y estudiantes?

\section{PLANTEAMIENTO DE LA HIPÓTESIS}

Hipótesis general:

El diseño actual de las aulas universitarias produce incomodidad y fatiga en docentes y estudiantes.

\section{Hipótesis específicas:}

1. La postura, el mobiliario actual, las condiciones ambientales y de seguridad desfavorables en las aulas universitarias provocan incomodidad y fatiga de docentes y estudiantes.

2. Las medidas antropométricas de docentes y estudiantes no son compatibles con el mobiliario actual generando incomodidad para la enseñanza y el aprendizaje universitario.

\section{Identificación de variables:}

\section{Variable independiente:}

Diseño de las aulas universitarias.

Indicadores:

- Postura del usuario

- Mobiliario

- Condiciones

- Ambientales

- Seguridad

- Variable dependiente:

- Reducción de Incomodidad y fatiga. 


\section{MÉTODOS Y RESULTADOS}

\subsection{Método y tipo de investigación}

El tipo de investigación utilizado es experimental, se aplicó también la descriptiva y explicativa. En ese sentido, se determinan las causas percibidas por los usuarios como las que generan la incomodidad y la fatiga y se explica si estas son relevantes para considerarlas dentro del diseño del aula, asimismo, se levanta información sobre las medidas antropométricas de docentes y estudiantes y se contrastan con las medidas del mobiliario a fin de determinar si estas guardan relación con las medidas de sus usuarios o no y dado el caso hacer las recomendaciones.

\section{Población de Estudio}

La población identificada estará compuesta por docentes, estudiantes y mobiliario de las aulas de la Facultad de Ingeniería Industrial de la UNMSM.

\section{Tamaño y Selección de la Muestra}

\section{Muestra de docentes:}

$$
n=\frac{\left(N \times z^{2} \times p \times q\right)}{\left(d^{2} \times(N-1)\right)+\left(z^{2} \times p x q\right)}
$$

$\mathrm{N}=55$ docentes nombrados en la FII

- $Z^{2}=1.96^{2}$ (si la seguridad es del 95\%)

- $p$ y $q=0.5$

- $\quad d=$ precisión (en este caso deseamos un $6 \%)$.

$$
\begin{gathered}
\mathrm{n}=\left(55 \times 1,96^{2} \times 0,5^{*} 0,5\right) /(0,062 \times(55- \\
1))+\left(1,96^{2} \times 0,5 \times 0,5\right)
\end{gathered}
$$

Aplicando la fórmula:

$$
n=45.6=46 \text { docentes }
$$

\section{Muestra de estudiantes:}

Se tomó como población conocida el total de estudiantes matriculados en los últimos 8 semestres (periodo 2007-1 al 2012-2) agrupados por bases de ingreso, para tener la certeza de que los estudiantes encuestados pertenezcan a bases cuya permanencia en la universidad es distinta. El promedio de estudiantes matriculados $\mathrm{N}=1133$, entonces:

- $N=1133$ estudiantes

- $Z^{2}=1.96^{2}$ (si la confiabilidad es del $95 \%$ )

- p y q = proporción esperada 0.5
- $\mathrm{d}=$ precisión (en este caso deseamos un $5.15 \%)$.

Aplicando la formula anterior:

$n=274.59$ entonces $n=275$ estudiantes

En la Tabla 1 se muestra la selección del grupo de estudiantes.

Tabla 1. Muestra de estudiantes de la EAP Ing. Industrial tomada según base de ingreso

\begin{tabular}{|c|c|c|c|}
\hline Base & 2012 & 2011 & 2010 \\
\hline muestra & 30 & 50 & 50 \\
\hline
\end{tabular}

\begin{tabular}{|c|c|c|c|}
\hline 2009 & 2008 & 2007 & Total \\
\hline 50 & 50 & 45 & 275 \\
\hline
\end{tabular}

Fuente: Elaboración propia.

\section{Muestra para nedidas antropométricas}

En el caso de los docentes la mayor parte del tiempo permanecen de pie sin embargo existe una postura alternativa que es sentado por lo que las medidas consideradas para ellos están indicadas en la figura 1 con los números 1 al 10 donde el $\mathrm{N}^{\circ} 1$ corresponde a la estatura, $\mathrm{N}^{\circ} 2$ altura ojossuelo, de pie, $\mathrm{N}^{\circ} 3$ altura codo-suelo, de pie, $\mathrm{N}^{\circ}$ 4 altura poplítea, $N^{\circ} 5$ altura ojos-asiento, $N^{\circ} 6$ ancho de hombros, $\mathrm{N}^{\circ} 7$ ancho de caderas, $\mathrm{N}^{\circ} 8$ altura codo-asiento, № 9 distancia sacro poplítea, $\mathrm{N}^{\circ} 10$ distancia sacro-rotula, y están descritas en el acápite Medidas antropométricas el punto 2.3 Marco Conceptual.

Figura 1. Medidas Antropométricas.

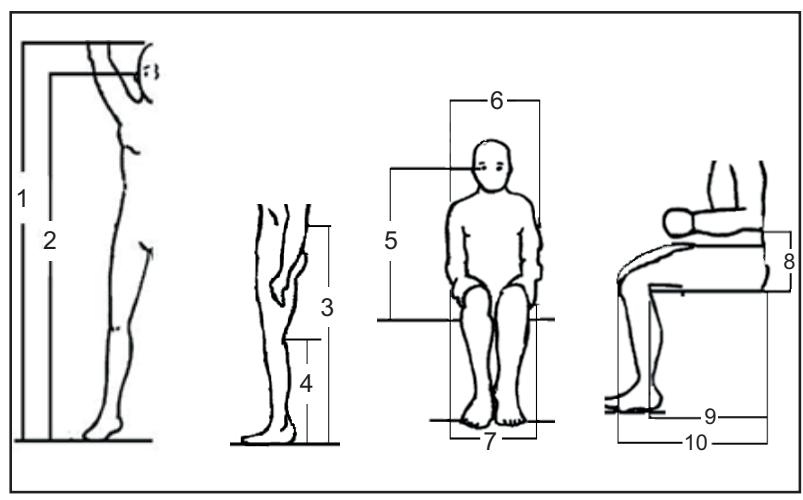

Fuente: imágenes adaptadas de internet. Recuperado de http://www.retosport.com/II-Semana-de-Antropometria-en-laUACHO. (Enero 2012) 
En tanto que para los estudiantes, cuya postura predominante es sentada, se tomaron las medidas indicadas en la figura 1 con los números $4,7,8,9$ y 10.

La muestra de docentes sexo masculino tomada fue de $n=44$, esto arroja una confiabilidad de $95 \%$ y una precisión del $7 \%$, debido a la resistencia de los mismos a ser medidos. Las docentes sexo femenino son menos del $2.5 \%$ y no se consideró.

La Tabla 2 incluye los percentiles 5 y 95 considerados como medidas mínima y máxima a considerar en el diseño del mobiliario.

En el caso de los estudiantes se tomó las medidas por sexo para lo cual se determinó que el porcentaje de estudiantes sexo femenino que se matricularon en los años 2007 al 2012 fue en promedio del $30 \%$. Es por ello que la muestra tomada consideró dicho porcentaje. La tabla 3 , muestra las medidas antropométricas consideradas a los estudiantes por sexo en los percentiles 5 y 95 .

Las dimensiones recogidas tanto a docentes como estudiantes se realizaron sin zapatos y en cuanto a la ropa utilizada se hizo en el caso de docentes con camisa y pantalón y a los estudiantes con polo manga corta y jean que es el mínimo de prendas de vestir aceptado por la muestra.

\section{Prueba de Hipótesis:}

\section{Hipótesis 1:}

Se aplicó la prueba chi cuadrada para el análisis de datos cualitativos a fin de encontrar si existe una relación entre la frecuencia en cada categoría de la variable dependiente (Comodidad o Incomodidad) y la variable independiente (postura, mobiliario, condiciones ambientales, seguridad). En caso de no encontrar una diferencia en la preferencia esto llevaría a pensar que la variable no es percibida como

Tabla 2. Medidas antropométricas de docentes

\begin{tabular}{|c|c|c|}
\hline Medida antropométrica docentes & Percentil 5 & Percentil 95 \\
\hline Estatura & 158.5 & 174.5 \\
\hline Altura a los ojos desde suelo & 146.5 & 163.5 \\
\hline Altura codo suelo & 98 & 112 \\
\hline Altura poplítea & 44 & 54 \\
\hline Altura ojos asiento & 70 & 83 \\
\hline Ancho de hombros & 45 & 50 \\
\hline Ancho de caderas & 37 & 48 \\
\hline Altura codo asiento & 19 & 27 \\
\hline Distancia sacro poplítea & 38 & 47 \\
\hline Distancia sacro-rotula & 51 & 61 \\
\hline
\end{tabular}

Fuente: Elaboración propia.

Tabla 3. Medidas antropométricas de estudiantes por sexo

\begin{tabular}{|c|c|c|c|c|c|}
\hline$N^{*}$ & $\begin{array}{c}\text { Medida antropométrica } \\
\text { estudiantes }\end{array}$ & \multicolumn{2}{|c|}{ masculino } & \multicolumn{2}{c|}{ femenino } \\
\cline { 3 - 6 } & & Percentil 5 & Percentil 95 & Percentil 5 & Percentil 95 \\
\hline 4 & Altura poplítea & 45 & 58 & 41 & 55 \\
\hline 6 & Ancho de hombros & 40 & 48 & 35 & 45 \\
\hline 7 & Ancho de caderas & 31 & 41 & 28 & 42 \\
\hline 8 & Altura codo asiento & 19 & 29 & 18 & 29 \\
\hline 9 & Distancia sacro poplítea & 39 & 52 & 39 & 47 \\
\hline 10 & Distancia sacro-rotula & 50 & 62 & 47 & 58 \\
\hline
\end{tabular}

Fuente: Elaboración propia. 
Tabla 4. Resultados de la aplicación de la prueba chi cuadrada a los indicadores postura, mobiliario, condiciones ambientales y seguridad para los docentes de la FII (UNMSM), con el software Minitab $\circledast$

\begin{tabular}{|c|c|c|c|c|}
\hline & Indicador & valor chi cuadrada & Grados de libertad & Valor critico \\
\hline 1 & Postura & 27,777 & 2 & 9,2104 \\
\hline 2 & Mobiliario & 32,894 & 4 & 13,2767 \\
\hline 3 & $\begin{array}{c}\text { Condiciones } \\
\text { Ambientales }\end{array}$ & 29,354 & 3 & 11,3449 \\
\hline 4 & Seguridad & 4,483 & 1 & 6,6349 \\
\hline
\end{tabular}

Fuente: Elaboración propia.

Tabla 5. Resultados de la aplicación de la prueba chi cuadrada a los indicadores mobiliario, condiciones ambientales y seguridad para los estudiantes de la FII (UNMSM), con el software Minitab®

\begin{tabular}{|c|c|c|c|c|}
\hline & Factor & valor chi cuadrada & Grados de libertad & Valor crítico \\
\hline 1. & Mobiliario:(carpeta) & & & \\
\hline & Mobiliario (respaldo) & 245,774 & 4 & 13,2767 \\
\hline & Mobiliario (asiento) & 258,373 & 4 & 13,2767 \\
\hline 3 & $\begin{array}{c}\text { Condiciones } \\
\text { Ambientales }\end{array}$ & 41,616 & 3 & 11,3449 \\
\hline 4 & Seguridad & 9,107 & 1 & 6,6349 \\
\hline
\end{tabular}

Fuente: Elaboración propia.

Tabla 6. Medidas del mobiliario y el resultado en la comodidad de docentes y estudiantes

\begin{tabular}{|c|l|l|}
\hline \multicolumn{1}{|c|}{ Docentes } & \multicolumn{1}{|c|}{ Resultado } \\
\hline Atril & $\begin{array}{l}\text { No hay coincidencia con la medida 3 del } \\
\text { percentil 5 -altura al codo de pie. }\end{array}$ & Incomodidad para el apoyo de brazos y lectura \\
\hline Mueble de cómputo & $\begin{array}{l}\text { No hay coincidencia con la medida 6 y el } \\
\text { percentil 95, ancho de caderas. } \\
\text { No hay coincidencia con la medida 10 y el } \\
\text { percentil 95, distancia sacro-poplitea. }\end{array}$ & $\begin{array}{l}\text { Incomodidad. No ingresan ambas piernas, } \\
\text { profundidad. }\end{array}$ \\
\hline Pizarra & $\begin{array}{l}\text { Es mayor a la medida 1 del percentil 95, } \\
\text { estatura }\end{array}$ & Cómodo. La dimensión es adecuada. \\
\hline Silla & $\begin{array}{l}\text { No coincide con la medida 4 del percentil 5, } \\
\text { altura poplítea }\end{array}$ & Incómodo. Los pies no llegan al suelo. \\
\hline Asiento carpeta & $\begin{array}{l}\text { No coincide con la medida 4 del percentil 5, } \\
\text { sexo femenino, altura poplítea. }\end{array}$ & $\begin{array}{l}\text { Incómodo. Los pies no llegan al suelo con una } \\
\text { diferencia de 4 cm. }\end{array}$ \\
\hline Respaldo carpeta & $\begin{array}{l}\text { Es menor en 9 cm con la medida 6 del percentil } \\
95 \text { y 1 cm con el percentil 5, sexo masculino } \\
\text { ancho de hombros }\end{array}$ & $\begin{array}{l}\text { El tamaño del respaldo es insuficiente para el } \\
\text { soporte de la espalda. }\end{array}$ \\
\hline Tablero & $\begin{array}{l}\text { Es mayor a la medida 6 del percentil 95, sexo } \\
\text { masculino ancho de hombros }\end{array}$ & Es adecuado para la tarea de escritura. \\
\hline $\begin{array}{c}\text { Distancia asiento- } \\
\text { tablero }\end{array}$ & $\begin{array}{l}\text { Es mayor a la medida } 8 \text { del percentil 5 sexo } \\
\text { masculino y femenino altura codo asiento. }\end{array}$ & $\begin{array}{l}\text { Es la distancia mínima adecuada para la tarea } \\
\text { de escritura y el espacio de las piernas. }\end{array}$ \\
\hline
\end{tabular}


$\mathrm{H}^{\circ}=$ No existe relación entre la sensación de confort (cómodo/incomodo) por los docentes respecto del indicador postura adoptada.

$\mathrm{H} 1=\mathrm{Si}$ existe relación entre la sensación de confort (cómodo/incomodo) por los docentes respecto del indicador postura adoptada.

Las tablas 4 y 5 muestran los resultados de la aplicación del software Minitab ${ }^{\circledR}$ para la prueba chi cuadrada. En el caso de docentes se puede concluir que si existe relación en la sensación de confort para los indicadores postura, mobiliario y condiciones ambientales y no así para la seguridad. En el caso de los estudiantes si existe relación en la sensación de confort en todos los indicadores evaluados.

\section{Hipótesis 2}

Se contrastó las medidas del mobiliario utilizado tanto por el docente como por el estudiante con sus datos antropométricos obtenidos. El resultado que se muestra en la tabla 6.

En resúmen existen aspectos que son desfavorables a la comodidad de los estudiantes.

\section{RESULTADOS}

\section{Efectos en la comodidad}

La Figura 2 muestra las variables que representan más del $50 \%$ de incomodidad para los docentes, se hizo la

Figura 2. Porcentaje de incomodidad mayor al $50 \%$ percibida por los docentes.

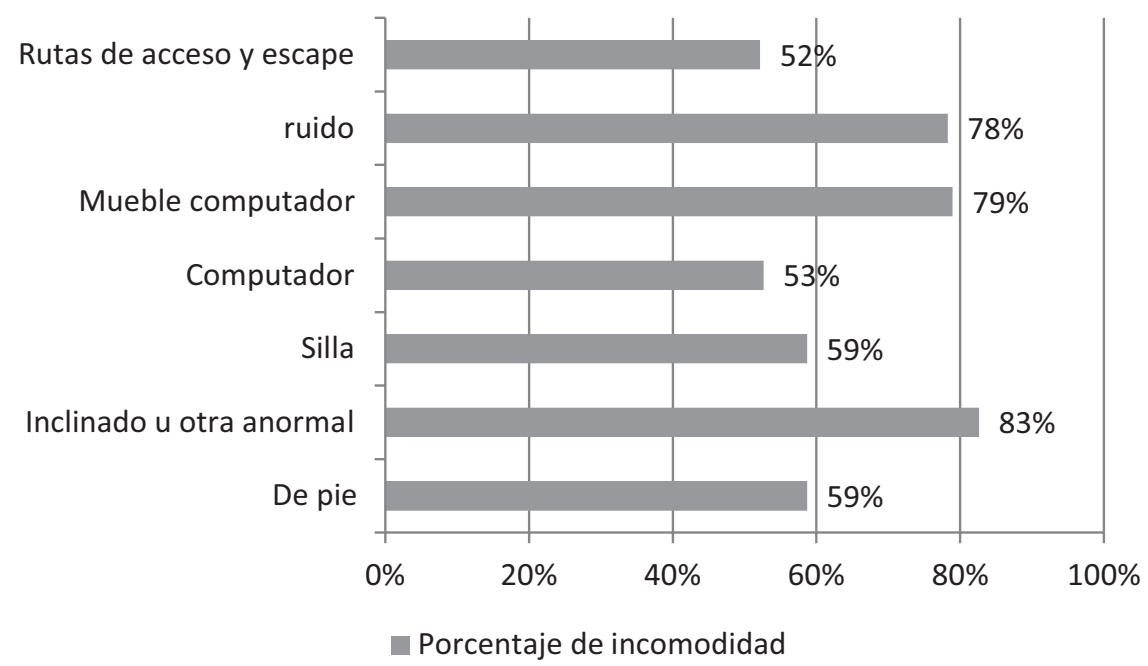

Fuente: Docentes de la Fll de la UNMSM. Elaboración propia.

Figura 3. Porcentaje de respuesta como "incomodidad" dada por docentes debido a la ubicación, diseño y altura del mobiliario utilizado.

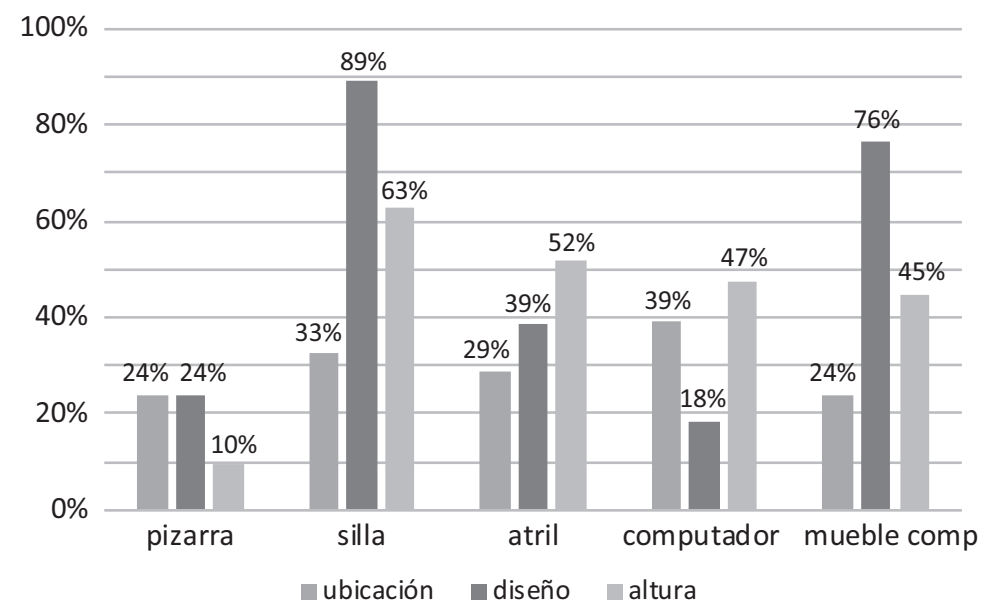

Fuente: Elaboración propia. 
aclaración a los docentes para que sus respuestas se den de acuerdo del mobiliario que el docente utilizaba para que pueda proporcionar su grado de comodidad respecto al uso de los mismos, para esos casos la muestra $n$ varía. Las cuatro percibidas como las más incomodas son la postura inclinada o anormal, el mueble del computador, el ruido y la silla.

Mantener una postura forzada o no neutral puede producir dolor y fatiga disminuyendo la capacidad de trabajo y por tanto la productividad. Es importante conocer por tanto, la o las causas que obligan al docente a mantener una postura inclinada por lo que se les preguntó si la incomodidad que sentían con el mobiliario utilizado estaba relacionada con la ubicación, el diseño o la altura del mobiliario. En la figura 3 se observa el porcentaje de respuesta de lo cual se puede concluir que la postura inclinada es lo que más afecta su comodidad y se debe al diseño de la silla y del mueble de cómputo. Por lo cual habría que modificar ambos en primer lugar para lograr un mayor confort en los docentes.

De la encuesta formulada a los estudiantes resultó que la opinión del $90 \%$ de los mismos indica que se siente cómodo con la postura sentada, mientras que sus apreciaciones de incomodidad sobre la carpeta son la dureza del material del asiento y la forma del respaldo, lo que se observa en la figura 4.

\section{Efectos en la fatiga}

El $41 \%$ y $36 \%$ de los docentes y estudiantes respectivamente indicó sentir fatiga al final de la jornada. Por lo que para determinar cómo se manifiesta y en qué medida se preguntó si existía algún malestar o dolor físico en base al mapa de Corlett y Bishop de diferentes partes del cuerpo encontrándose para los docentes que las principales causas de malestar se encontraban en la espalda media $(20 \%)$, las piernas $(11 \%)$ y los pies (11\%) y las causas de dolor: los pies $(26 \%)$. Los estudiantes manifestaron que las principales causas de malestar y dolor estaban en las sentaderas $(47 \%$ y $2 \%$ malestar y dolor respectivamente), muslos (29\%malestar), espalda media $(24 \%$ y $3 \%$ ) y manos y muñecas ( $3 \%$ dolor) además se les solicitó que eligieran entre los indicadores de postura, mobiliario, iluminación, ruido, temperatura u otra causa la que pudiera considerar la razón principal, en la figura 5 se muestran las más representativas.

Figura 4. Porcentaje de respuesta dada por estudiantes como efecto de "incomodidad" debido al asiento y respaldo de la carpeta utilizada.

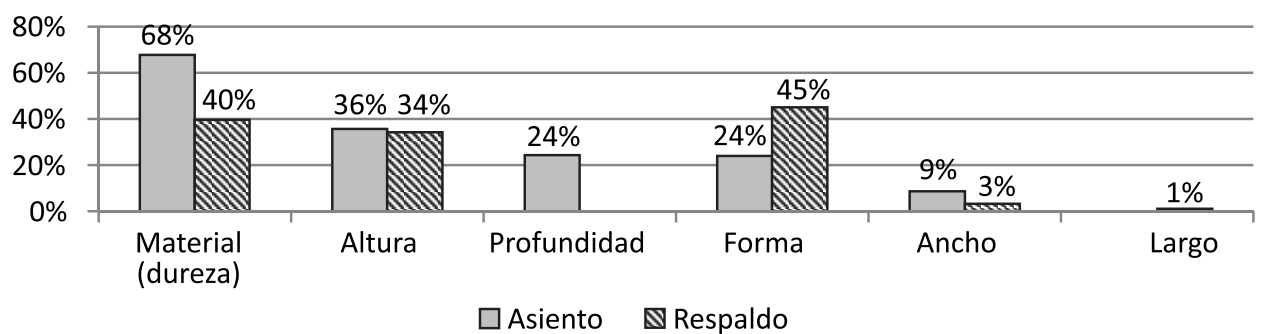

Fuente: Elaboración propia.

Figura 5. Principales causas de Malestar o dolor (fatiga) en docentes y estudiantes.

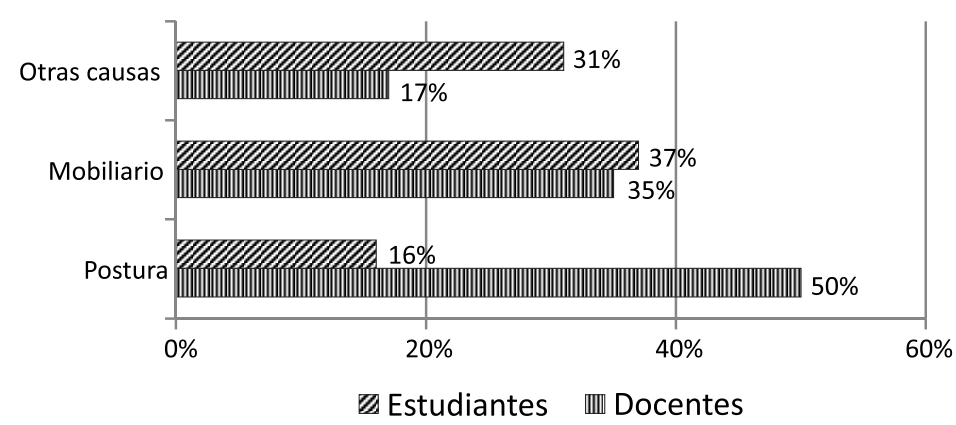

Fuente: Elaboración propia. 


\section{PROPUESTA SOBRE EL DISEÑO ERGONÓMI- CO DE LAS AULAS}

\subsection{Diseño ergonómico del mobiliario}

La presente investigación ha tomado los elementos encontrados causantes de la incomodidad para poder sugerir un diseño que considere los requerimientos de docentes y estudiantes y sus dimensiones antropométricas los mismos que se muestran en la figura 6 .

\subsection{Diseño ergonómico del aula universitaria}

El diseño del aula universitaria propuesto señala las consideraciones ergonómicas necesarias en: el mobiliario, las condiciones ambientales y la seguridad.

En cuanto al mobiliario, en el punto 6.1 se mostraron dos propuestas: una para ser considerada como mobiliario que puede usar el docente y otro para la carpeta del estudiante, ambos diseños han sido elaborados con criterio ergonómico considerando los indicadores investigados que para este criterio son postura adoptada y medidas antropométricas tomada a la muestra de docentes y estudiantes.

Respecto a las condiciones ambientales se ha tomado en cuenta, la normatividad para efectuar recomendaciones puesto que las sugerencias obtenidas de los usuarios sobre la temperatura y el ruido, son parámetros que dependen en muchos casos de la subjetividad individual, por ello, de acuerdo a la normas, los valores límites de contaminación acústica varían según los reglamentos municipales pero de forma general, el nivel de ruido aceptable en los centros de enseñanza varía entre $50 \mathrm{~dB}$ para el horario diurno y no debe superar $40 \mathrm{~dB}$ para el horario nocturno.

Figura 6. Mobiliario para el docente y el estudiante universitario.
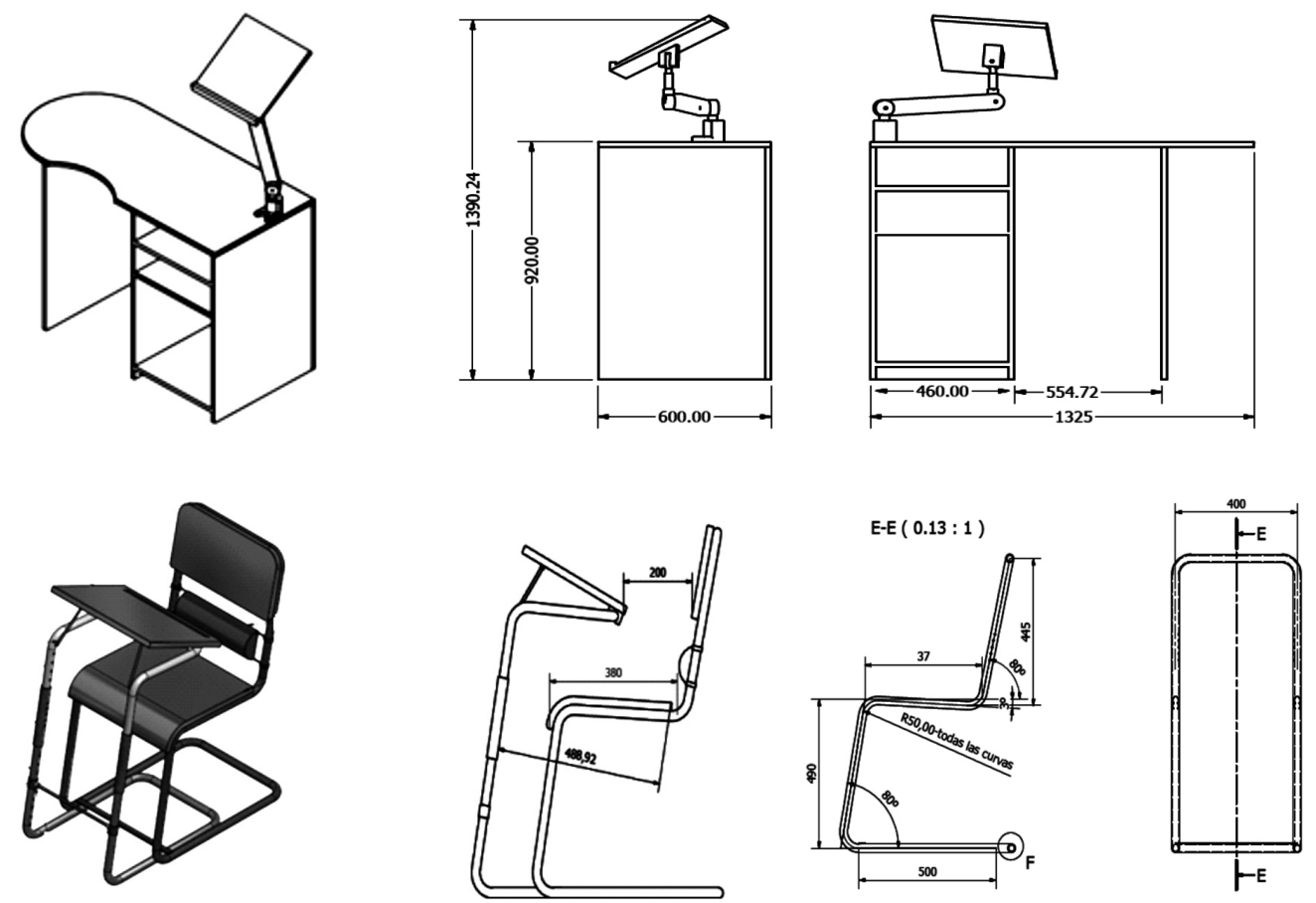

Fuente: Elaboración propia.

Tabla 7. Intervalo Óptimo de temperatura en actividades sedentarias

\begin{tabular}{|c|c|c|c|c|}
\hline Estación & $\begin{array}{c}\text { Temperatura } \\
\text { Mínima }\end{array}$ & $\begin{array}{c}\text { Temperatura } \\
\text { Máximo }\end{array}$ & Humedad relativa & Velocidad del aire \\
\hline Invierno & $20^{\circ} \mathrm{C}$ & $21^{\circ} \mathrm{C}$ & $<30 \%$ & $<0,2 \mathrm{~m} / \mathrm{s}$ \\
\hline Verano & $20^{\circ} \mathrm{C}$ & $24^{\circ} \mathrm{C}$ & $40-60 \%$ & $0,2 \mathrm{~m} / \mathrm{s}$ \\
\hline
\end{tabular}

Fuente: Tomado de Mondelo. "Confort y Estrés Térmico". 
Según la norma técnica de prevención española, Ntp 503: Confort acústico: el ruido en oficinas, dada por el Ministerio de trabajo de España, los niveles de ruido en $\mathrm{db}(\mathrm{a})$ para los centros de enseñanza deben ser: Aulas $40 \mathrm{db}(A)$, Salas de lectura 35 $\mathrm{db}(\mathrm{A})$ y Zonas comunes $50 \mathrm{db}(\mathrm{A})$.

En un estudio realizado por Edvard por encargo del Ministerio de Energía y Minas (Perú-1997), Dirección General de Asuntos Ambientales, el ruido tiende a interferir en la comunicación con el auditorio, donde el discurso es más importante. La percepción del discurso tiene especial importancia en los salones de clase o auditorios de conferencias, y en situaciones donde los oyentes tienen dificultades de audición. Para una distancia de $1 \mathrm{~m}$. del hablante al oyente es conocido que se puede tener un nivel de fondo de hasta:

- $45 \mathrm{~dB}$, un discurso relajante es $100 \%$ comprensible

- $55 \mathrm{~dB}$, un discurso puede ser entendido claramente

- $65 \mathrm{~dB}$, un discurso hablado con un mayor esfuerzo vocal puede entenderse.

Para reducir el ruido que procede del exterior se deben disponer ventanas dobles. En cuanto al ruido interior, se debe controlar el ruido que se hace en los pasillos y escaleras, tener cuidado al mover las mesas y las sillas.

En lo que respecta a la iluminación recomendada para las aulas de acuerdo a la reglamentación vigente se considera apropiado que la iluminación para las aulas sea de 300 lux.

En términos generales, los colores al interior de las aulas, deberán ser de tonos claros para contribuir con la mejor iluminación interior, dado que existirá un mejor reflejo de la luz al incidir sobre las superficies.

En un estudio realizado por el Ministerio de Educación se dan algunas recomendaciones para los paramentos que conforman los ambientes interiores de las edificaciones educativas como las siguientes:

Techos: la superficie de un techo debe ser lo más blanca posible, con un factor de reflexión de .75 ó $75 \%$, porque así reflejará la luz de manera difusa, disipando la oscuridad y reduciendo los brillos de otras superficies. A ello se añade el ahorro en iluminación artificial.

Paredes y suelos: las superficies de las paredes situadas a nivel de los ojos pueden provocar deslumbramiento, los colores pálidos con factores de reflexión del 50 al $75 \%$ suelen ser adecuados para las paredes. Aunque las pinturas brillantes tienden a durar más tiempo que los colores mate, son más reflectantes. Por consiguiente, las paredes deberán tener un acabado mate o semibrillante. Los acabados de los suelos deberán ser de colores ligeramente más oscuros que las paredes y los techos para evitar brillos. El factor de reflexión de los suelos debe oscilar entre el 20 y el $25 \%$.

Mobiliario y/o Equipo: cualquiera de las superficies de trabajo, ya sean carpetas, mesas de trabajo, tableros y maquinaria, etc. deberán tener factores de reflexión de entre 20 y $40 \%$. Los equipos deberán tener un acabado duradero de un color puro - gris o marrones claros-y el material no deberá ser brillante.

En cuanto a la temperatura, la Norma6 Básica de Ergonomía señala que los niveles de temperatura considerados como máximo a partir del cual se genera estrés térmico en términos de WBGT (temperatura globo bulbo húmedo) es de $30,5^{\circ} \mathrm{C}$, para una categoría de trabajo leve $75 \%$ de trabajo y $25 \%$ de descanso, dado que tanto el docente como el estudiante universitario dicta o recibe clases no más de 6 horas al día en promedio. Sin embargo, no puede mencionarse este valor como una temperatura que brinde comodidad por lo que, al no encontrar en esta norma valores de temperatura para el confort térmico, se recomienda como los valores de temperatura óptimos (nivel de confort) lo señalado en Mondelo y otros (2001), lo que se muestra en la tabla 7.

Los valores señalados como límites para el nivel de ruido $40 \mathrm{db}$, iluminación recomendada $300 \mathrm{~lx}$ y condiciones de temperatura para invierno y verano en promedio se consideran las condiciones ambientales favorables de forma que permitan impartir las clases en un estado de comodidad.

En el aspecto de Seguridad debe considerarse que las aulas deben contar con 2 puertas que sirvan para una evacuación rápida para casos de sismo, de igual forma el espacio para el aula debe considerarse un área de 1,30 m2 por estudiante-carpeta.

Teniendo en cuenta las consideraciones expuestas, se puede lograr un aula universitaria que brinde comodidad y reduzca la fatiga en sus usuarios.

\section{CONCLUSIONES Y RECOMENDACIONES}

\subsection{Conclusiones}

1. Los aspectos ergonómicos que más han influido en la incomodidad de los docentes en el aula universitaria son la postura de pie e inclinada, 
primero, porque su actividad lo obliga muchas veces a estar de pie y segundo, porque ni el mueble de computo ni el atril se adaptan a su estatura y dimensiones, el otro aspecto es el mobiliario pues su diseño y dimensiones no se adaptan al desarrollo de la labor docente. En el caso de los estudiantes, su principal causa de incomodidad es solo el mobiliario, principalmente el asiento de la carpeta y específicamente debido a la dureza del material.

2. En menor medida docentes y estudiantes han valorado las condiciones ambientales de iluminación, ruido, temperatura y ventilación como un aspecto ergonómico que afecte su comodidad sin embargo se puede señalar que de estas el ruido es el que en mayor grado les afecta. En tanto que la seguridad es un aspecto que docentes y estudiantes no toman en cuenta para señalar que las aulas son cómodas o incomodas.

3. La fatiga se ha relacionado con el grado de malestar o dolor que sienten al final de la jornada encontrándose en los docentes que está relacionada con el dolor pies y de garganta después de clases, en cuanto a los estudiantes se encontró que está en función al malestar en las sentaderas y en menor medida en los muslos y espalda media.

4. Las medidas del mobiliario del aula no guardan relación con las medidas antropométricas de los docentes y estudiantes.

\subsection{Recomendaciones}

1. El mobiliario del aula universitaria debe poder regularse o adaptarse para satisfacer las diferentes medidas de los usuarios y evitar que adopten posturas forzadas.

2. Para aliviar el dolor de pies en los docentes se recomienda colocar, en la plataforma donde se ubica, alfombra u otro material que amortigüe la tensión al caminar y dé comodidad a los pies, además de ser un aislante térmico que brinda mayor calidez que los pisos duros como los de cemento, sin contar que esto brinda la sensación de sentirse valorado. En cuanto al color se sugiere el azul u otra tonalidad neutral que produzca un ambiente tranquilo y relajado.

3. El asiento del docente y estudiante debe contar con un apoyo a la región lumbar para corregir la postura. En cuanto al material del asiento la carpeta requiere un material menos rígido que la melanina como por ejemplo la formica.
4. En el caso del docente, en lugar del asiento acolchado, se recomienda el uso de telas impermeables de trama esterilizadas (vinilo) para evitar la traspiración y el depósito de micro organismos.

5. Si bien es cierto, los docentes y estudiantes no han dado mayor importancia a las condiciones ambientales, el ruido generado en los cambios de hora debe minimizarse, con reglamentos al respecto y charlas a los estudiantes sobre la contaminación de ruido y sus perjuicios, asimismo las aulas no son acústicas y requieren mayor esfuerzo del docente para ser escuchados por la audiencia, en cuanto a la temperatura y la ventilación es preciso regularlas no solo para comodidad del aula sino también por motivos de salud, por ello son aspectos que hay que tener en cuenta para mejorarlos.

\section{REFERENCIAS BIBLIOGRÁFICAS}

[1] Edvard Falch (M.Sc.) por encargo de Ministerio de Energía y Minas, República del Perú, Dirección General de Asuntos Ambientales 1997. Manejo de problemas de ruido. Recuperado el 09/09/2013 de: http://www. minem.gob.pe/minem/archivos/file/DGAAM/ guias/ruidominera.pdf

[2] Esteve, J. M. (2005). Bienestar y salud docente. Revista PRELAC. Proyecto Regional de Educación para América Latina y el Caribe. UNESCO. Publicación electrónica recuperada de: http://unesdoc.unesco.org/ images/0014/001446/144666s.pdf

[3] Gómez-Cano M. Ruido (2007). Ruido: Evaluación y Acondicionamiento Ergonómico. Publicación electrónica del Instituto Nacional de seguridad e Higiene en el trabajo C/Torrelaguna, 73.28027. Madrid. Recuperado de http://www. insht.es/InshtWeb/Contenidos/Documentacion/ Aplicaciones/ficherosCuestionarios/naranja.pdf

[4] Mondelo, P. Gregori, E. Barrau P. (2001). Ergonomía 3. Diseño de puestos de trabajo. México D.F. AlfaOmega Grupo Editor.

[5] Vives V. (2005). El Movimiento Pedagógico del colegio de Docentes como una recuperación del rol docente y de la autoestima profesional. Tesis de maestría con publicación electrónica. Universidad de Chile. Santiago, Chile. Recuperado de: http://www.tesis.uchile.cl/tesis/ uchile/2005/vives_v/sources/vives_v.pdf 